\title{
Comment on: Rheology of an Ionic Liquid with Variable Carreau Exponent: A Full Picture by Molecular Simulation with Experimental Contribution, by Nicolas Voeltzel, Philippe Vergne, Nicolas Fillot, Nathalie Bouscharain, Laurent Joly, Tribology Letters (2016) 64:25
}

\section{H. A. Spikes ${ }^{1}$}

Received: 9 November 2016/Accepted: 11 April 2017/Published online: 28 April 2017

(c) The Author(s) 2017. This article is an open access publication

In a recent paper, Voeltzel et al. describe a non-equilibrium molecular dynamic (NEMD) study of the rheological properties of a single ionic liquid over range of temperatures, pressures and shear rates [1]. They also measure the low shear rate viscosity of the same fluid experimentally over a similar temperature and pressure range (Fig. 1).

The authors present their NEMD results in $\log$ (viscosity) versus $\log$ (shear rate) form. This shows classical shearthinning behaviour, in which, for data obtained at a given temperature and pressure, the viscosity is initially constant at low shear rate but decreases above a critical shear rate in an apparently linear fashion on a log/log plot, as shown in Fig. 2 (reproduced as Fig. 1). Interestingly, when the authors apply time-temperature superposition to convert all of their viscosity and shear rate data to reduced values based on a reference state of pressure $=0.1 \mathrm{MPa}$ and temperature $=303 \mathrm{~K}$, all of the data collapse onto a single master curve as shown in Fig. 3 (reproduced as Fig. 2).

The authors then best fit the Carreau rheological equation to this collapsed curve;

$\eta *=\eta_{\mathrm{oR}}\left(1+\left(t_{\mathrm{relR}} \dot{\gamma}^{*}\right)^{2}\right)^{\left(N_{R}-1\right) / 2}$

where $\eta^{*}$ is the reduced viscosity, $\dot{\gamma}^{*}$ is the reduced shear rate, $\eta_{\mathrm{oR}}$ is the low shear rate viscosity at the reference state, and the two disposable Carreau constants are a relaxation time $t_{\text {relR }}$ and a power index $N_{R}$, both at the reference state. The authors also treat $\eta_{\mathrm{oR}}$ as a disposable constant and their best fit, shown as a dashed line in Fig. 3, is respectable with relaxation time $t_{\text {relR }}=0.612 \mathrm{~ns}$ and

\footnotetext{
$\triangle$ H. A. Spikes

h.spikes@imperial.ac.uk

1 Imperial College London, London, UK
}

$N_{R}=0.213$. However, as noted by the authors, it requires $\eta_{\mathrm{oR}}=29.9 \mathrm{mPa} \mathrm{s}$, which is less than the measured low shear rate reference viscosity of $32.5 \mathrm{mPa}$ s. It also deviates significantly at very high shear rates. When the authors apply Eq. 1 to each separate sets of raw viscosity/shear rate data at a given temperature and pressure, they also get good fits but are concerned to note that the two fit constants at the various conditions, $t_{\text {rel }}$ and $N$, appear intrinsically and inexplicably correlated, as they illustrate in Fig. 9 [1].

I suggest that the discrepancies and the correlation between $t_{\text {rel }}$ and $N$ noted in their paper result from Voeltzel et al.'s choice of the Carreau equation. There are many rheological equations to describe shear-thinning behaviour of liquids, but the two most widely employed in tribology are the Carreau (or its extension Carreau-Yasuda) and the Eyring equation [2]. Eyring's equation is usually written in terms of shear stress, $\tau$

$\tau=\tau_{\mathrm{E}} \sinh ^{-1}\left(\frac{\eta_{o} \dot{\gamma}}{\tau_{\mathrm{E}}}\right)$

where $\tau_{\mathrm{E}}$ is the Eyring stress that marks the onset of shear thinning. However, it is easily converted to a similar form to Eq. 1 by using $\tau=\eta \dot{\gamma}, \tau_{\mathrm{E}}=\eta_{o} \dot{\gamma}_{E}$ and $t_{\text {relE }}=1 / \dot{\gamma}_{E}$, where $\dot{\gamma}_{E}$ is a critical shear rate. In reduced variable form, the Eyring equation then becomes

$\eta^{*}=\frac{\eta_{\mathrm{oR}}}{t_{\mathrm{relER}} \dot{\gamma}^{*}} \sinh ^{-1}\left(t_{\mathrm{relER}} \dot{\gamma}^{*}\right)$

where $t_{\text {relER }}$ is a relaxation time at the reference state and is similar but not identical to the Carreau one.

Figure 3 shows Fig. 2 on which is superimposed a solid line showing the "best fit" of Eyring's Eq. 3, taking $\eta_{\mathrm{oR}}$ to be Voeltzel et al.'s best fit value of $29.9 \mathrm{mPa}$. This is obtained using a value of $t_{\text {relER }}=1.33 \mathrm{~ns}\left(\tau_{\mathrm{ER}}=22.5\right.$ MPa). There is clearly a very good match to the data, at 


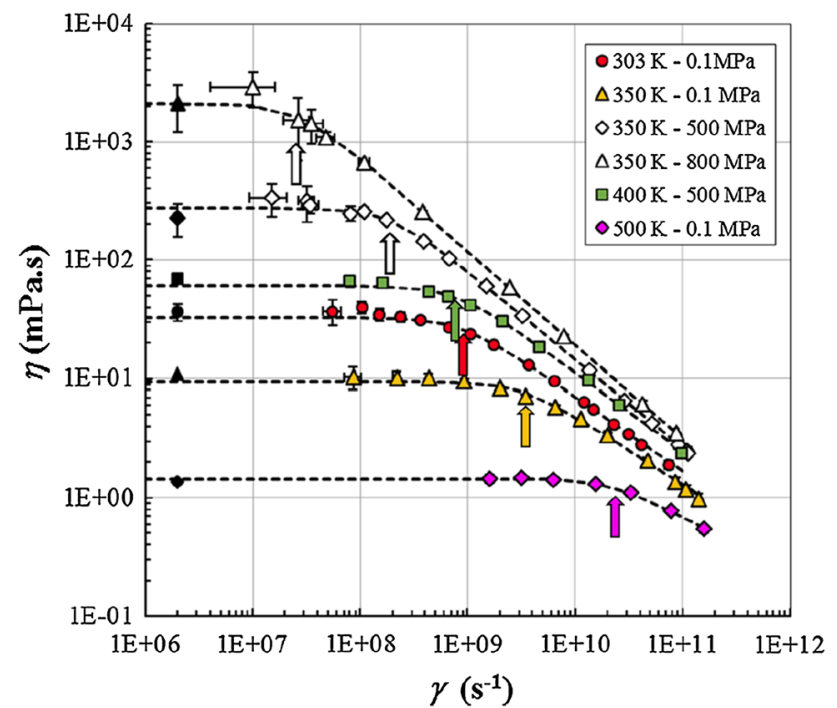

Fig. 1 Variation of NEMD viscosity against shear rate at various pressures and temperatures for an ionic liquid. Reproduced from ref. [1]

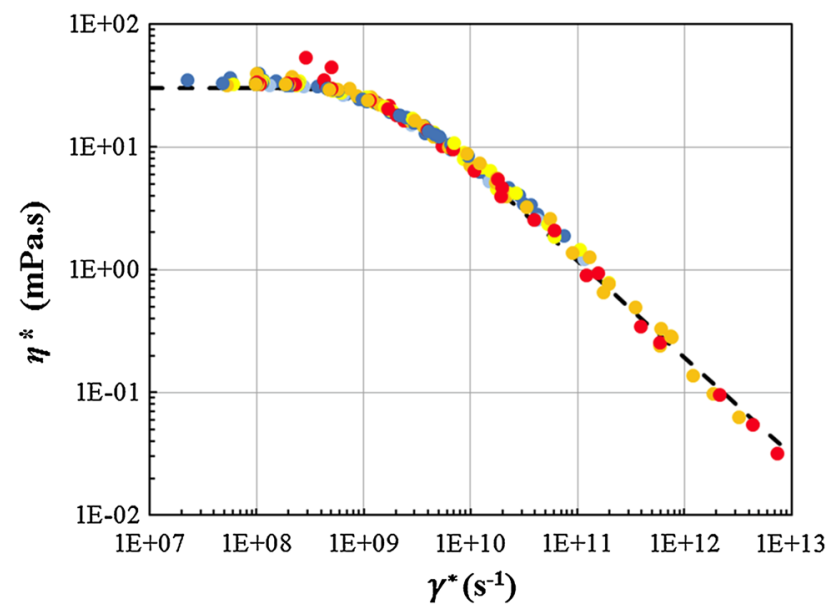

Fig. 2 Reduced viscosity versus reduced shear rate for an ionic liquid. Reproduced from ref. [1]

least as good as the Carreau fit. The Carreau equation is constrained to converge to a straight line at high strain rate on such a $\log / \log$ plot, while the Eyring one is not. This means that the latter can follow the NEMD data more closely at high strain rate. Figure 4 shows a very similar plot, but here $\eta_{o R}$ is taken to be its measured reference value of $32.5 \mathrm{mPa} \mathrm{s}$ and $t_{\text {relER }}=1.38 \mathrm{~ns}$. This appears to give an even better fit over the whole reduced shear rate range. These two fits were obtained simply by adjusting $t_{\text {relER }}$ to match the data by eye-no doubt even closer fits could have been obtained by mathematical optimisation where the numerical NEMD data available.

It is important to note that these extremely good fits were obtained with a rheological equation that has just one disposable fit constant $t_{\text {rele }}$. It follows that if an equation

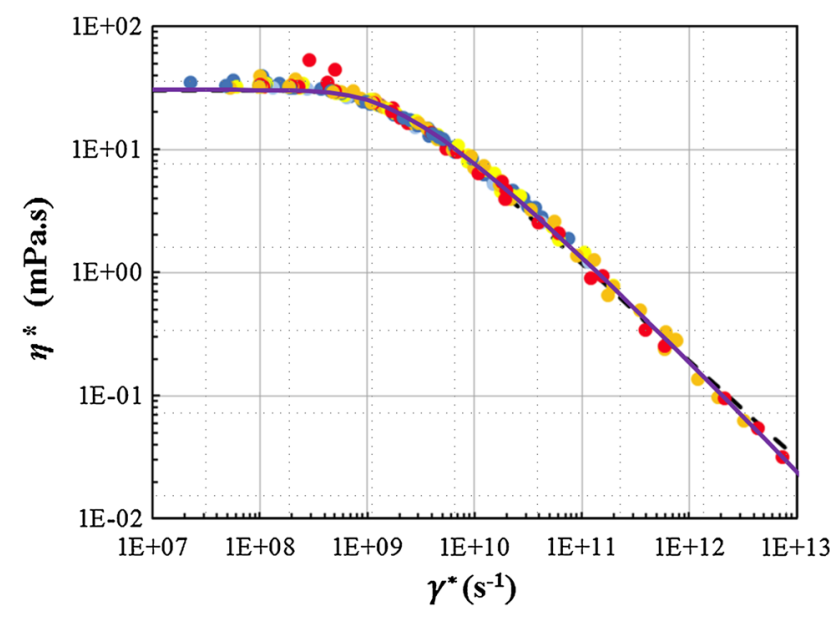

Fig. 3 Plot showing fit of Eyring equation to NEMD reduced viscosity-reduced shear rate data

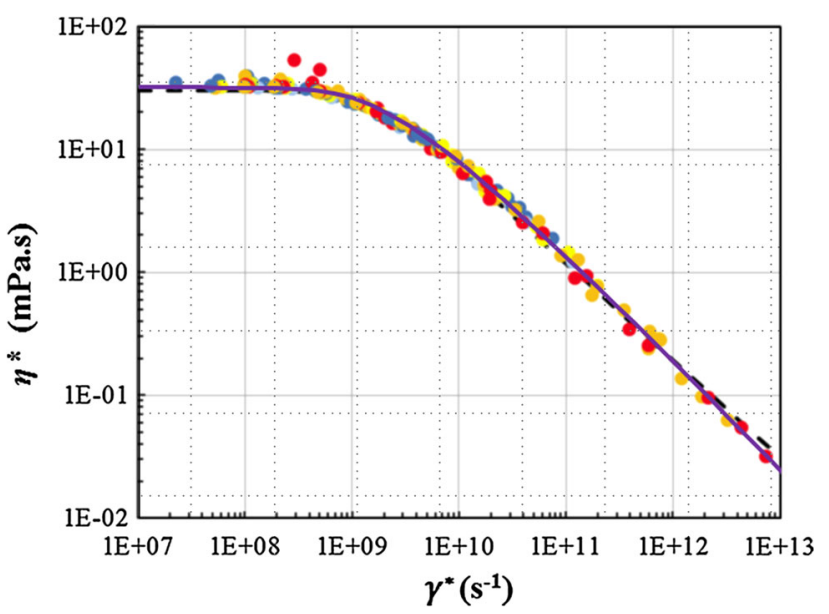

Fig. 4 Plot showing fit of Eyring equation to NEMD reduced viscosity-reduced shear rate data using $\eta_{\mathrm{oR}}=32.5 \mathrm{mPa} \mathrm{s}$

with two disposable constants such as Carreau also fits the data, its two constants must be inherently related, as found by Voeltzel et al. The nature of this relationship will be determined solely by the mathematical form of the two equations.

The above indicates that the single-constant Eyring rheological equation fits the NEMD data at least as well as the two-constant Carreau equation, and it is a pity that Voeltzel et al. did not test it alongside the latter since there is some controversy concerning the best rheological model to describe shear thinning of simple liquids at high shear stress [2-4]. Both equations originate from physical models: the Carreau model from a network forming/breaking model of polymer flow and the Eyring model from a stressactivated molecular flow model for simple liquids, and it is arguable that an ionic liquid is closer to a simple liquid than a polymer. 


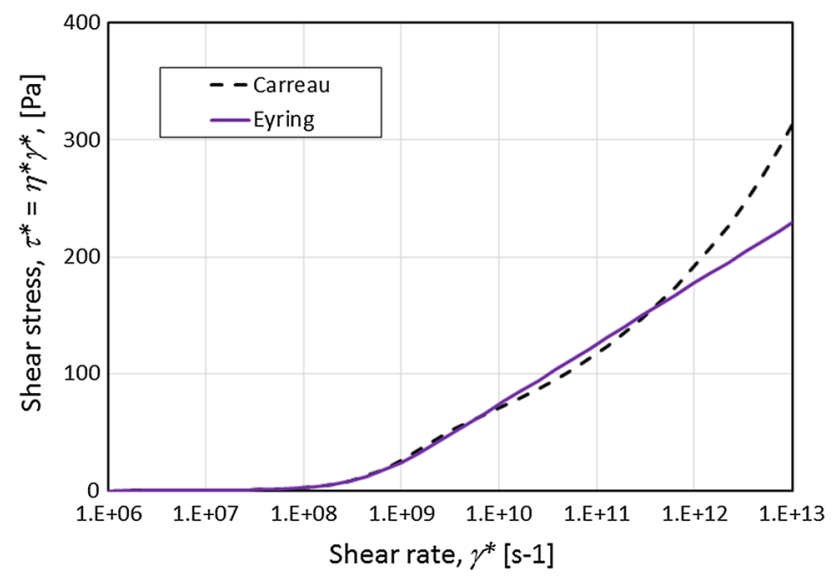

Fig. 5 Comparison of Carreau and Eyring fits when plotted in shear stress versus $\log$ (strain rate) form, showing divergence at high strain rate

One way to explore the issue further would be to examine the data in a different form. It is perhaps unfortunate that Voeltzel et al. used a $\log / \log$ representation of their viscosity/shear rate data. Such plots tend to promote the appearance of linearity at the expense of resolution at the extremities of the plot. An alternative way to represent the data is to plot shear stress, calculated from viscosity $\mathrm{x}$ strain rate, versus $\log$ (strain rate). This linear/log form of plot reveals differences in behaviour at high strain rates much more clearly that the $\log / \log$ form. Figure 5 compares Voeltzel et al.'s Carreau fit with the Eyring fit shown in Fig. 3. There is evidently a large divergence at reduced shear rates above $10^{11} \mathrm{~s}^{-1}$ that is obscured in the $\log / \log$ plot of Fig. 1. I thus urge the authors and future researchers to test their NEMD data in such a plotted form to see whether the Eyring or Carreau models best fits the data and, if possible, to extend their reduced shear rate range above $10^{13} \mathrm{~s}^{-1}$.
Of course, the fact that a particular rheological equation fits experimental or NEMD data closely does not per se validate the equation. This should be evident from the above, where it is seen that two quite different models fit the data closely over most of the range studied. However, if carried out over a very wide range of stress conditions and used to test more than one rheological equation, the NEMD approach is potentially a powerful tool for comparing and exploring the validity of shear-thinning equations for simple liquids.

Open Access This article is distributed under the terms of the Creative Commons Attribution 4.0 International License (http://crea tivecommons.org/licenses/by/4.0/), which permits unrestricted use, distribution, and reproduction in any medium, provided you give appropriate credit to the original author(s) and the source, provide a link to the Creative Commons license, and indicate if changes were made.

\section{References}

1. Voeltzel, N., Vergne, P., Fillot, N., Bouscharain, N., Joly, L.: Rheology of an ionic liquid with variable Carreau exponent: a full picture by molecular simulation with experimental contribution. Tribol. Lett. 64, 25 (2016)

2. Spikes, H.A., Zhang, J.: History, origins and prediction of elastohydrodynamic friction. Tribol. Lett. 56, 1-25 (2014)

3. Bair, S., Vergne, P., Kumar, P., Poll, G., Krupka, I., Hartl, M., Habchi, W., Larsson, R.: Comment on "History, origins and prediction of elastohydrodynamic friction" by Spikes and Jie. Tribol. Lett. 58, 1-8 (2015)

4. Spikes, H.A., Zhang, J.: Reply to the Comment by Scott Bair, Philippe Vergne, Punit Kumar, Gerhard Poll, Ivan Krupka, Martin Hartl, Wassim Habchi, Roland Larson on "History, origins and prediction of elastohydrodynamic friction" by Spikes and Jie in Tribology Letters. Tribol. Lett. 58, 17 (2015) 